\title{
Trends in Urgency of Emergency Department Visits among Those with and without Multiple Chronic Conditions, 2007-2012
}

\begin{abstract}
Objectives: The objective of this study was to examine overall trends in Emergency Department (ED) visits among adults with and without multiple chronic conditions (MCC) by urgency of visit (nonurgent, urgent, primary care sensitive) and across relevant population subgroups.
\end{abstract}

Methods: Using data from the Nationwide Emergency Department Sample (NEDS) for 2007-2012 maintained by the Healthcare Cost and Utilization Project (HCUP), we examined trends in ED visits among adults who were diagnosed as having no chronic conditions (OCC), one chronic condition (1CC), and MCC based on the Clinical Classification Software codes. Separate trend analyses were conducted for ED visits that were classified as urgent, nonurgent, and primary care sensitive (PCS) based on the New York University (NYU) ED Algorithm. Trend analyses were also conducted for several population subgroups: age, sex, metropolitan hospital status, Census Region, insurance status, and median income quartiles by ZIP code.

Results: Between 2007 and 2012, the overall percentage change in nonurgent ED visits made by adults with OCC, 1CC and MCC were $8.05 \%, 22.50 \%$ and $35.36 \%$ respectively ( $<<0.001$ for all categories). Primary Care Sensitive (PCS) ED visits among adults with OCC, 1CC and MCC increased by $6.53 \%, 15.49 \%$ and $16.53 \%$, respectively, from 2007-2012 ( $p<0.001$ for all categories). Among adults with OCC, 1CC and MCC, different patterns of growth trends were found for urgent, nonurgent, and PCS ED visits across analyzed population subgroups.

Conclusion: The results show that chronic conditions, especially multiple chronic conditions, played a role in the long-term growth of ED visits in general and in nonurgent and primary care sensitive (PCS) ED visits. The increases in nonurgent and PCS visits highlight the importance, as well as opportunities, to reduce potentially avoidable ED visits and unnecessary health care costs, especially as the MCC population is expected to grow in the years ahead.

Keywords: Emergency department use; Emergency department trends; Multiple chronic conditions; NYU algorithm; HCUP; NEDS

\section{Paige Powell , Xinhua Yu, Oluwaseyi Isehunwa and Cyril F Chang}

The University of Memphis School of Public Health, USA

\section{Corresponding author: Powell MP \\ 戸 mppowell@memphis.edu}

Assistant Professor of Health Systems Management and Policy, University of Memphis School of Public Health, 124 Robison Hall, Memphis ,TN, USA-381520003.

Tel: 901-678-2883

Fax: 901-678-0383

Citation: Powell MP, Yu X, Isehunwa O, et al. Trends in Urgency of Emergency Department Visits among Those with and without Multiple Chronic Conditions, 20072012. J Hosp Med Manage. 2016, 2:2.

Received: November 11, 2016; Accepted: December 01, 2016; Published: December 05, 2016

\section{Introduction}

Over the past few decades in the United States, the number of Emergency Department (ED) visits and the prevalence of multiple chronic conditions (MCC) have both been rising steadily [1-3]. Cross-sectional studies have shown that ED use is highest among blacks and Medicaid patients with chronic ambulatory care sensitive (ACS) conditions [1]. Patients in fair or poor health, or with chronic conditions are higher utilizers of the ED than those in excellent to good health or those without chronic conditions [2]. The most frequent users of ED services tend to be those with multiple chronic conditions and repeated hospitalizations [3]. There has also been a heightened focus in research on ED visits that are considered nonurgent in that they require no 
immediate medical attention at the time of ED treatment and can potentially be avoided by the receipt of effective ambulatory care in the community setting. Uninsured, younger patients, blacks, females, Medicaid, and self-paying patients are more likely to use the ED for nonurgent conditions [4,5]. In addition, having public insurance was associated with a higher probability of ED visits being nonurgent or PCS relative to having private insurance or no insurance [6]. To our knowledge, however, previous work has not examined whether MCCs affect urgent and nonurgent visits differently. Although much has been learned, gaps in the understanding of the reasons for the high and rising levels of hospital spending associated with ED visits and the potential for overcrowding still exist. Our study will fill a gap in the ED literature by examining recent nonurgent ED trends with a focus on patients' chronic conditions at the time of their ED visits.

Estimates of the cost of emergency care in the US range from $2 \%$ of National Health Expenditures ( $\$ 48.3$ billion) to $10 \%$ ( $\$ 300$ billion), although the most reliable estimates are 5-6\% (\$150$\$ 180$ billion) [7]. The National Priorities Partnership estimated that over half of all ED visits were nonurgent or potentially avoidable [8]. Internationally, hospital emergency departments in the U.S. see a much larger percentage of nonurgent patients than most other industrialized nations ( $21 \%$ compared to $6 \%$ in Germany and $11 \%$ in the UK) [9]. Reducing wasteful spending in the ED could save the U.S. $\$ 38$ billion in emergency spending and potentially reduce costs in other areas as well.

\section{Data and Methods}

We used data from the 2007-2012 Nationwide Emergency Department Sample (NEDS) developed for the Healthcare Cost and Utilization Project (HCUP) by the Agency for Healthcare Research and Quality. To develop this dataset, HCUP selected a stratified, single stage, cluster sample of hospitals based on census region, trauma center designation, urban/rural location, teaching status, and ownership to obtain about 20 percent of all hospital ED visits within each stratum. Once a hospital was sampled, all discharges from that hospital's ED were included in the database. The sample frame varied by year depending on states' participation in HCUP. As of 2012, the sampling universe consisted of hospital based EDs from 950 hospitals in 30 states, and about 30 million ED visits were included in the sample. Hospital based post-stratification weights, developed by HCUP, were used to generalize the results to the total number of hospital based ED visits annually (134 million in 2012) [10]. The NEDS data also included diagnosis and procedure codes, expected payer information, hospital characteristics, ED and hospital charges, and limited demographic data on the patient. We limited our analyses to visits by patients who were 45 years or older, both because chronic disease affects less than $20 \%$ of adults $18-44$ and to be consistent with other research studies that used the same age cut-off $[7,8]$. We divided age into three categories: $45-$ $64,65-79$, and $80+$ years old. Census regions were classified as Northeast, South, Midwest, and West. We collapsed the HCUP urban-rural designation into a dichotomous variable measuring metropolitan ("large" and "small" metropolitans) versus nonmetropolitan areas ("micropolitan" and "not metropolitan or micropolitan"). Health insurance is categorized as public (Medicare and Medicaid), private, self-pay/uninsured, and other. HCUP also included quartiles of the median annual income of patients by ZIP code: 1) \$1-\$38,999; (2) \$39,000-\$47,999; (3) $\$ 48,000-\$ 62,999$; and (4) $\$ 63,000$ or more.

To be consistent with previous MCC studies, we used the common chronic conditions classified in the National Health Interview Survey (NHIS) based on Clinical Classification Software (CCS) codes. They included hypertension (CCS codes: 98,99), coronary heart disease $(100,101)$, stroke $(109-112)$, diabetes $(49,50)$, cancer, excluding non-melanoma skin cancer (11-43), arthritis $(203,203)$, hepatitis (6), chronic kidney disease (158), asthma (128), and chronic obstructive pulmonary disease (127) [10-14]. We defined MCC as having at least two of the above 10 CCs.

We identified the urgency level of each ED visit using the empirically validated NYU ED algorithm developed originally by researchers at New York University who abstracted and reviewed detailed patient information of a sample of almost 6,000 patient records by a panel of emergency physicians in order to determine the urgent nature of the visits [15-17]. To determine whether the ED visit was urgent or nonurgent based on the above classification, we followed the examples of Ballard and colleagues [18] and Tsai, Chen and Liang [17] (Table 1).

We applied the NEDS-provided hospital based survey weights for all analyses. The weighted frequencies estimated all hospital based ED visits including both hospitalized and not hospitalized ED visits. The percentages of growth rates were calculated by dividing the difference of frequencies between baseline year 2007 and each of the follow-up years by the baseline-year frequency, similar to the statistical methods used in HCUP publications [11]. To examine the trends of ED visits by MCC, we tested the statistical significance of long-term trends using $\chi^{2}$ trend analysis, similar to performing a linear regression on the changing rates. The interaction term between time and CC status in the linear models examined whether the trends among CC status differed significantly or not. Nonlinearity of the trend was judged visually based on the trend plots. In addition, due to the short duration of our study, we did not perform age adjustment; rather, the results were stratified by age groups, similar to those reported in HCUP. All the analyses were performed using SAS 9.4 (Cary, $\mathrm{NC}$ ). The statistical significance level was set to 0.001 to account for multiple comparison issues. This study was classified as "not human subjects research" by the IRB.

\section{Results}

As shown in Table 2, urgent ED visits for those without any chronic conditions declined $0.64 \%$, while those with 1 CC increased $14.55 \%$ and those with MCC increased $23.66 \%$. Nonurgent visits, as shown in Table 3, increased at faster rates across all three chronic condition groups, with visits by patients with OCC, 1CC and MCC increasing by $8.05 \%, 22.50 \%$, and $35.36 \%$, respectively. Similarly, Table 4 shows that PCS ED visits increased $6.53 \%$ for those with OCC, compared to $15.49 \%$ for those with $1 \mathrm{CC}$, and $16.53 \%$ for those with MCC. We present the results for visits by 
Table 1. New York University Algorithm Definition and Examples and Study Variables.

\begin{tabular}{|c|c|c|}
\hline NYU Classification & Definition & Examples \\
\hline Non-Emergent (ne) & Immediate care not required within 12 hours & sore throat, stuffy nose \\
\hline Emergent/Primary Care Treatable (epct) & $\begin{array}{l}\text { ED care needed within } 12 \text { hours but could be treated } \\
\text { effectively in a primary care setting }\end{array}$ & $\begin{array}{l}\text { lasting fever, other flu-like } \\
\text { symptoms }\end{array}$ \\
\hline $\begin{array}{l}\text { Emergent-ED Care Needed-Preventable/ } \\
\text { Avoidable (edcnpa) }\end{array}$ & $\begin{array}{c}\text { ED care needed but could be avoided if effective and } \\
\text { timely care was received before }\end{array}$ & Pneumonia, asthma exacerbation \\
\hline $\begin{array}{c}\text { Emergent-ED Care Needed-Not Preventable/ } \\
\text { Avoidable (edcnnpa) }\end{array}$ & $\begin{array}{c}\text { ED care truly required given patient's condition and no } \\
\text { ambulatory care could have prevented visit }\end{array}$ & appendicitis, stroke, heart attack \\
\hline Study Variable & Definition & \\
\hline Urgent & edcnnpa $>0.5+$ injury & \\
\hline Non-urgent & ne + epct $>0.5$ & \\
\hline Primary Care Sensitive (PCS) & ne + epct $>0.5+$ edcnpa $>0.5$ & \\
\hline
\end{tabular}

*Each ED visit in the study sample was given a probability and assigned into one of four ED groups that were summed up to 1 (or $100 \%$ ). Visits were also classified as 'injury', 'mental illness' and 'alcohol or substance abuse'. We excluded from the study all ED visits that were not classified or classified as mental illness, or alcohol or substance abuse following the instructions of the NYU ED Algorithm.

Table 2. Number and Percent Change of Urgent ED visits by chronic condition status between 2007 and 2012. 1, 2, 3, 4, 5, 6

\begin{tabular}{|c|c|c|c|c|c|c|c|c|c|}
\hline & \multicolumn{3}{|c|}{ OCC } & \multicolumn{3}{|c|}{$1 \mathrm{CC}$} & \multicolumn{3}{|c|}{ MCC } \\
\hline & 2007 & 2012 & \%change & 2007 & 2012 & \%change & 2007 & 2012 & \%change \\
\hline Total & $7,833,902$ & $7,783,875$ & -0.64 & $3,589,649$ & $4,111,941$ & 14.55 & $4,370,347$ & $5,404,250$ & 23.66 \\
\hline \multicolumn{10}{|l|}{ Age } \\
\hline $45-64$ & $5,449,378$ & $5,434,227$ & -0.28 & $1,789,198$ & $2,151,429$ & 20.25 & $1,519,413$ & $2,005,115$ & 31.97 \\
\hline $65-79$ & $1,455,339$ & $1,485,983$ & 2.11 & 999,426 & $1,113,357$ & 11.40 & $1,570,602$ & $1,903,986$ & 21.23 \\
\hline $80+$ & 929,187 & 863,666 & -7.05 & 801,026 & 847,156 & 5.76 & $1,280,334$ & $1,495,150$ & 16.78 \\
\hline \multicolumn{10}{|l|}{ Payer } \\
\hline Public & $2,988,539$ & $3,324,158$ & 11.23 & $2,002,821$ & $2,380,511$ & 18.86 & $3,177,221$ & $4,027,754$ & 26.77 \\
\hline Commercial & $3,184,229$ & $2,777,510$ & -12.77 & $1,114,631$ & $1,152,615$ & 3.41 & 868,920 & 950,353 & 9.37 \\
\hline Self-pay/no insurance & $1,009,020$ & $1,062,486$ & 5.30 & 304,090 & 368,364 & 21.14 & 203,435 & 257,211 & 26.43 \\
\hline Other & 652,116 & 619,723 & -4.97 & 168,109 & 210,453 & 25.19 & 120,773 & 168,934 & 39.88 \\
\hline \multicolumn{10}{|l|}{ Sex } \\
\hline Male & $3,661,270$ & $3,601,081$ & -1.64 & $1,547,135$ & $1,786,093$ & 15.45 & $1,992,967$ & $2,511,381$ & 26.01 \\
\hline Female & $4,170,114$ & $4,182,158$ & 0.29 & $2,040,908$ & $2,325,627$ & 13.95 & $2,376,200$ & $2,892,759$ & 21.74 \\
\hline \multicolumn{10}{|l|}{ Hospital region } \\
\hline Northeast & $1,792,924$ & $1,740,586$ & -2.92 & 640,792 & 751,497 & 17.28 & 757,743 & 907,962 & 19.82 \\
\hline Midwest & $1,851,423$ & $1,740,549$ & -5.99 & 835,286 & 933,455 & 11.75 & $1,070,724$ & $1,272,294$ & 18.83 \\
\hline South & $2,785,964$ & $2,900,073$ & 4.10 & $1,531,141$ & $1,655,977$ & 8.15 & $1,860,523$ & $2,221,275$ & 19.39 \\
\hline West & $1,403,593$ & $1,402,670$ & -0.07 & 582,431 & 771,013 & 32.38 & 681,359 & $1,002,721$ & 47.16 \\
\hline \multicolumn{10}{|l|}{ Metro status } \\
\hline Metropolitan & $5,337,256$ & $5,679,581$ & 6.41 & $2,506,570$ & $3,165,444$ & 26.29 & $3,124,659$ & $4,258,591$ & 36.29 \\
\hline Non-metropolitan & $2,496,647$ & $2,104,295$ & -15.72 & $1,083,080$ & 946,498 & -12.61 & $1,245,688$ & $1,145,660$ & -8.03 \\
\hline \multicolumn{10}{|l|}{ Zip code income quartile } \\
\hline Q1 & $2,088,244$ & $2,153,324$ & 3.12 & 999,645 & $1,143,774$ & 14.42 & $1,295,162$ & $1,623,641$ & 25.36 \\
\hline Q2 & $1,966,591$ & $1,922,017$ & -2.27 & 922,885 & $1,006,754$ & 9.09 & $1,140,920$ & $1,345,874$ & 17.96 \\
\hline Q3 & $1,090,242$ & $1,814,600$ & 66.44 & 865,636 & 959,548 & 10.85 & $1,040,300$ & $1,243,335$ & 19.52 \\
\hline Q4 & $1,711,048$ & $1,709,570$ & -0.09 & 712,973 & 915,517 & 28.41 & 797,331 & $1,090,242$ & 36.74 \\
\hline
\end{tabular}

${ }^{1}$ Source: Agency for Healthcare Research and Quality (AHRQ), Center for Delivery, Organization, and Markets, Healthcare Cost and Utilization Project (HCUP), Nationwide Emergency Department Sample (NEDS), 2007 and 2012.

${ }^{2}$ Chronic conditions and Clinical Classification Software Codes: hypertension $(98,99)$, coronary heart disease $(100,101)$, stroke (109-112), diabetes $(49,50)$, cancer, excluding non-melanoma skin cancer (11-43), arthritis $(203,203)$, hepatitis (6), chronic kidney disease (158), asthma (128), and chronic obstructive pulmonary disease (COPD) (127).

${ }^{3}$ All ED visits with a probability of "Emergent-ED Care Needed-Not Preventable/Avoidable (ednncpa)" $>0.5$ or classified as "injury" were classified "urgent." Those with combined probabilities of "non-emergent (ne)" + "emergent primary care treatable (epct)" >0.5 were classified as "nonurgent." "Primary care sensitive (PCS)" ED visits included all nonurgent visits defined above (i.e., cases with ne + epct $>0.5$ ) and those with a probability of "Emergent-ED Care Needed-Potentially Avoidable (edncpa)" >0.5.

${ }^{4}$ Public $=$ Medicare and Medicaid, Private $=$ Private, Self-pay/uninsured $=$ Self-pay, Other $=$ No charge and Other.

${ }^{5}$ Metropolitan = large metropolitan and small metropolitan, Non-metropolitan = micropolitan and not metropolitan or micropolitan.

${ }^{6}$ Median income of the zip code reported by quartile: $Q 1=\$ 1-\$ 38,999, Q 2=\$ 39,000-\$ 47,999, Q 3=\$ 48,000-\$ 62,999, Q 4=\$ 63,000$ or more. 
Table 3. Number and Percent Change of Nonurgent ED visits by chronic condition status between 2007 and 2012. 1,2,3,4,5, 6

\begin{tabular}{|c|c|c|c|c|c|c|c|c|c|}
\hline & 2007 & 2012 & \%change & 2007 & 2012 & \%change & 2007 & 2012 & \%change \\
\hline Total & $6,200,877$ & $6,700,321$ & 8.05 & $3,461,182$ & $4,239,920$ & 22.50 & $3,148,211$ & $4,261,484$ & 35.36 \\
\hline \multicolumn{10}{|l|}{ Age } \\
\hline $45-64$ & $4,555,557$ & $5,042,081$ & 10.68 & $2,014,462$ & $2,586,665$ & 28.40 & $1,410,984$ & $2,000,098$ & 41.75 \\
\hline $65-79$ & $1,072,741$ & $1,122,526$ & 4.64 & 888,694 & $1,033,029$ & 16.24 & $1,073,351$ & $1,404,065$ & 30.81 \\
\hline $80+$ & 572,580 & 535,715 & -6.44 & 558,027 & 620,226 & 11.15 & 663,877 & 857,322 & 29.14 \\
\hline \multicolumn{10}{|l|}{ Payer } \\
\hline Public & $2,635,932$ & $3,120,192$ & 18.37 & $1,872,220$ & $2,397,497$ & 28.06 & $2,181,677$ & $3,076,597$ & 41.02 \\
\hline Commercial & $2,346,438$ & $2,034,928$ & -13.28 & $1,055,483$ & $1,083,500$ & 2.65 & 670,222 & 744,317 & 11.06 \\
\hline Self-pay/no insurance & 959,753 & $1,194,827$ & 24.49 & 423,838 & 586,350 & 38.34 & 216,492 & 310,743 & 43.54 \\
\hline Other & 258,755 & 350,376 & 35.41 & 109,643 & 172,575 & 57.40 & 79,822 & 129,829 & 62.65 \\
\hline \multicolumn{10}{|l|}{ Sex } \\
\hline Male & $2,443,200$ & $2,629,652$ & 7.63 & $1,319,785$ & $1,617,760$ & 22.58 & $1,307,719$ & $1,772,163$ & 35.52 \\
\hline Female & $3,756,486$ & $4,070,428$ & 8.36 & $2,140,405$ & $2,622,019$ & 22.50 & $1,839,518$ & $2,489,216$ & 35.32 \\
\hline \multicolumn{10}{|l|}{ Hospital region } \\
\hline Northeast & $1,449,834$ & $1,485,611$ & 2.47 & 601,488 & 751,873 & 25.00 & 509,743 & 663,965 & 30.25 \\
\hline Midwest & $1,375,162$ & $1,481,699$ & 7.75 & 767,666 & 944,258 & 23.00 & 753,466 & $1,007,687$ & 33.74 \\
\hline South & $2,239,904$ & $2,535,400$ & 13.19 & $1,517,287$ & $1,783,870$ & 17.57 & $1,418,362$ & $1,826,657$ & 28.79 \\
\hline West & $1,135,978$ & $1,197,613$ & 5.43 & 574,743 & 759,921 & 32.22 & 466,643 & 763,177 & 63.55 \\
\hline \multicolumn{10}{|l|}{ Metro status } \\
\hline Metropolitan & $2,387,445$ & $4,968,012$ & 108.09 & $2,387,445$ & $3,244,998$ & 35.92 & $2,229,679$ & $3,332,666$ & 49.47 \\
\hline Non-metropolitan & $1,073,737$ & $1,732,309$ & 61.33 & $1,073,737$ & 994,923 & -7.34 & 918,533 & 928,819 & 1.12 \\
\hline \multicolumn{10}{|l|}{ Zip code income quartile } \\
\hline Q1 & $1,968,093$ & $2,213,589$ & 12.47 & $1,147,580$ & $1,414,887$ & 23.29 & $1,068,573$ & $1,471,940$ & 37.75 \\
\hline Q2 & $1,570,352$ & $1,673,779$ & 6.59 & 891,522 & $1,055,079$ & 18.35 & 827,229 & $1,067,007$ & 28.99 \\
\hline Q3 & $1,359,092$ & $1,483,943$ & 9.19 & 760,147 & 933,005 & 22.74 & 692,553 & 924,674 & 33.52 \\
\hline Q4 & $1,127,420$ & $1,166,978$ & 3.51 & 568,920 & 745,032 & 30.96 & 481,277 & 715,051 & 48.57 \\
\hline
\end{tabular}

${ }^{1}$ Source: Agency for Healthcare Research and Quality (AHRQ), Center for Delivery, Organization, and Markets, Healthcare Cost and Utilization Project (HCUP), Nationwide Emergency Department Sample (NEDS), 2007 and 2012.

${ }^{2}$ Chronic conditions and Clinical Classification Software Codes: hypertension $(98,99)$, coronary heart disease $(100,101)$, stroke $(109-112)$, diabetes $(49,50)$, cancer, excluding non-melanoma skin cancer (11-43), arthritis $(203,203)$, hepatitis (6), chronic kidney disease (158), asthma (128), and chronic obstructive pulmonary disease (COPD) (127).

${ }^{3}$ All ED visits with a probability of "Emergent-ED Care Needed-Not Preventable/Avoidable (ednncpa)" > 0.5 or classified as "injury" were classified "urgent." Those with combined probabilities of "non-emergent (ne)" + "emergent primary care treatable (epct)" $>0.5$ were classified as "nonurgent." "Primary care sensitive (PCS)" ED visits included all nonurgent visits defined above (i.e., cases with ne + epct $>0.5$ ) and those with a probability of "Emergent-ED Care Needed-Potentially Avoidable (edncpa)" >0.5.

${ }^{4}$ Public $=$ Medicare and Medicaid, Private $=$ Private, Self-pay/uninsured $=$ Self-pay, Other $=$ No charge and Other.

${ }^{5}$ Metropolitan = large metropolitan and small metropolitan, Non-metropolitan = micropolitan and not metropolitan or micropolitan.

${ }^{6}$ Median income of the zip code reported by quartile: $Q 1=\$ 1-\$ 38,999, Q 2=\$ 39,000-\$ 47,999, Q 3=\$ 48,000-\$ 62,999, Q 4=\$ 63,000$ or more.

subgroup across all urgency categories (Table 2: urgent, Table 3: non-urgent, Table 4: PCS). By age, the largest changes were for those 45-64 years old, while the oldest age group (80+ years old) had the slowest growth across all three categories of ED visits and all categories of chronic conditions. On the other hand, those 80 years and older with MCC had 16.78\%, 29.14\%, and $3.26 \%$ growth for urgent, nonurgent, and PCS ED visits, respectively. There were significant decreases between 2007 and 2012 in the rates of urgent, nonurgent, and PCS visits $(-7.05 \%,-6.44 \%$, and $-9.27 \%$, respectively) for those 80 years and older with no chronic conditions.

Among payer types, although urgent, nonurgent, and PCS visits increased for all chronic condition categories for those paid by public, self-pay, and other insurance types, visits paid by commercial insurance decreased significantly in all categories for those with OCC $(-12.77 \%,-13.28 \%$, and $-13.61 \%$, respectively). Also, the increase of urgent and nonurgent visits among commercial visits with $1 \mathrm{CC}$ and MCC were smaller than for those paid by either public insurance or self-pay status (Tables $\mathbf{2}$ and 3). The trends in PCS visits remained flat among commercially paid visits and 1 CC, and they exhibited a much smaller increase $(2.12 \%)$ among those with MCC and commercial insurance when compared with those paid for by other payer types (Table 4).

Fewer ED visits were made by males than by females across all three categories of chronic conditions and all three urgency types. Among the four geographic regions, urgent visits for OCC declined in all regions except the South. While nonurgent and PCS visits increased in all regions for OCC, the largest percent growth of urgent visits was also in the South. For both 1CC and MCC, ED visits increased across all urgency categories and 
all census regions, but the largest growth was in the West for urgent, nonurgent, and PCS visits.

We also found downward trends in urgent and PCS ED visits for non-metropolitan areas across almost all categories of chronic condition status, while there was a significant increase of nonurgent ED visits for those with OCC. However, urgent, nonurgent, and PCS visits increased significantly for those with MCC in metropolitan EDs, with the largest increase occurring for nonurgent visits. For those living in metropolitan areas with OCC, a surprising increase (108.09\%) of nonurgent visits was observed. For those with 1CC, a statistically significant decrease $(-53.65 \%)$ of PCS ED visits was observed.

Patients in the highest median income quartile by ZIP code had the largest increase of urgent, nonurgent, and PCS visits if they had 1 CC or MCC, compared with those with other income quartiles. Those with in the lowest income quartile had the second largest increase of all types of ED visits for those with 1CC and $\mathrm{MCC}$, while those in the second income quartile generally had the smallest increase of all types of ED visits. Meanwhile, those with OCC tended to have smaller increases or even slight decreases in ED visits, compared with those with 1CC and MCC.

To explore the patterns of growth of ED visits in detail, we present growth trend data for all three categories of ED visits in Figures 1 and 2. There was a steady increase in nonurgent visits from 2007-2012 across all three age groups for those with 1CC and MCC (Figure 1), and the difference in growth rates between them was significant $(p<0.001)$. Nonurgent visits for those with OCC remained steady until 2011, with an uptick in the percentage change occurring between 2011 and 2012. Similarly, for PCS visits, those with $1 \mathrm{CC}$ or MCC exhibited an increasing trend across all age groups, with the age group 45-64 years old showing the fastest growth rate. For those with $0 \mathrm{CC}$, there was a mild growth

Table 4. Number and Percent Change of primary care sensitive ED visits by chronic condition status between 2007 and $2012 .{ }^{1,2,3,4,5,6}$

\begin{tabular}{|c|c|c|c|c|c|c|c|c|c|}
\hline & \multicolumn{3}{|c|}{ OCC } & \multicolumn{3}{|c|}{$1 \mathrm{CC}$} & \multicolumn{3}{|c|}{ MCC } \\
\hline & 2007 & 2012 & \%change & 2007 & 2012 & \%change & 2007 & 2012 & \%change \\
\hline Total & $6,634,088$ & $7,067,531$ & 6.53 & $4,093,586$ & $4,727,491$ & 15.49 & $4,677,827$ & $5,450,948$ & 16.53 \\
\hline \multicolumn{10}{|l|}{ Age } \\
\hline $45-64$ & $4,817,414$ & $5,274,543$ & 9.49 & $2,290,575$ & $2,827,802$ & 23.45 & $1,874,689$ & $2,419,850$ & 29.08 \\
\hline $65-79$ & $1,160,040$ & $1,197,212$ & 3.20 & $1,068,941$ & $1,165,767$ & 9.06 & $1,639,656$ & $1,829,744$ & 11.59 \\
\hline $80+$ & 656,635 & 595,777 & -9.27 & 734,072 & 733,923 & -0.02 & $1,163,483$ & $1,201,375$ & 3.26 \\
\hline \multicolumn{10}{|l|}{ Payer } \\
\hline Public & $2,885,573$ & $3,331,049$ & 15.44 & $2,304,894$ & $2,725,742$ & 18.26 & $3,410,464$ & $4,026,252$ & 18.06 \\
\hline Commercial & $2,462,870$ & $2,127,656$ & -13.61 & $1,189,019$ & $1,181,803$ & -0.61 & 885,061 & 903,842 & 2.12 \\
\hline Self-pay/no insurance & $1,012,846$ & $1,244,501$ & 22.87 & 472,985 & 631,954 & 33.61 & 271,552 & 362,040 & 33.32 \\
\hline Other & 272,800 & 364,327 & 33.55 & 126,689 & 187,995 & 48.39 & 110,751 & 158,836 & 43.42 \\
\hline \multicolumn{10}{|l|}{ Sex } \\
\hline Male & $2,649,180$ & $2,793,314$ & 5.44 & $1,617,432$ & $1,844,930$ & 14.07 & $2,043,229$ & $2,356,145$ & 15.31 \\
\hline Female & $3,983,571$ & $4,273,970$ & 7.29 & $2,474,968$ & $2,882,396$ & 16.46 & $2,633,351$ & $3,094,706$ & 17.52 \\
\hline \multicolumn{10}{|l|}{ Hospital region } \\
\hline Northeast & $1,538,857$ & $1,555,915$ & 1.11 & 728,364 & 838,773 & 15.16 & 814,731 & 864,638 & 6.13 \\
\hline Midwest & $1,475,362$ & $1,570,532$ & 6.45 & 913,140 & $1,059,614$ & 16.04 & $1,106,670$ & $1,283,356$ & 15.97 \\
\hline South & $2,387,947$ & $2,669,965$ & 11.81 & $1,754,359$ & $1,971,450$ & 12.37 & $2,031,627$ & $2,320,227$ & 14.21 \\
\hline West & $1,231,923$ & $1,271,120$ & 3.18 & 697,724 & 857,656 & 22.92 & 724,800 & 982,748 & 35.59 \\
\hline \multicolumn{10}{|l|}{ Metro status } \\
\hline Metropolitan & $4,509,713$ & $5,221,978$ & 15.79 & $2,797,153$ & $1,296,434$ & -53.65 & $3,293,165$ & $4,217,781$ & 28.08 \\
\hline Non-metropolitan & $2,124,375$ & $1,845,553$ & -13.12 & $1,296,434$ & $1,139,793$ & -12.08 & $1,384,662$ & $1,233,188$ & -10.94 \\
\hline \multicolumn{10}{|l|}{ Zip code income quartile } \\
\hline Q1 & $2,106,647$ & $2,333,546$ & 10.77 & $1,353,400$ & $1,575,545$ & 16.41 & $1,570,724$ & $1,872,646$ & 19.22 \\
\hline Q2 & $1,681,989$ & $1,769,012$ & 5.17 & $1,059,847$ & $1,182,573$ & 11.58 & $1,224,527$ & $1,374,035$ & 12.21 \\
\hline Q3 & $1,451,037$ & $1,565,526$ & 7.89 & 895,869 & $1,039,717$ & 16.06 & $1,031,531$ & $1,181,418$ & 14.53 \\
\hline Q4 & $1,204,427$ & $1,227,678$ & 1.93 & 674,899 & 826,322 & 22.44 & 735,699 & 916,948 & 24.64 \\
\hline
\end{tabular}

${ }^{1}$ Source: Agency for Healthcare Research and Quality (AHRQ), Center for Delivery, Organization, and Markets, Healthcare Cost and Utilization Project (HCUP), Nationwide Emergency Department Sample (NEDS), 2007 and 2012.

${ }^{2}$ Chronic conditions and Clinical Classification Software Codes: hypertension (98,99), coronary heart disease $(100,101)$, stroke $(109-112)$, diabetes $(49,50)$, cancer, excluding non-melanoma skin cancer (11-43), arthritis $(203,203)$, hepatitis (6), chronic kidney disease (158), asthma (128), and chronic obstructive pulmonary disease (COPD) (127).

${ }^{3}$ All ED visits with a probability of "Emergent-ED Care Needed-Not Preventable/Avoidable (ednncpa)" > 0.5 or classified as "injury" were classified "urgent." Those with combined probabilities of "non-emergent (ne)" + "emergent primary care treatable (epct)" > 0.5 were classified as "nonurgent." "Primary care sensitive (PCS)" ED visits included all nonurgent visits defined above (i.e., cases with ne + epct > 0.5) and those with a probability of "Emergent-ED Care Needed-Potentially Avoidable (edncpa)" > 0.5 .

${ }^{4}$ Public =Medicare and Medicaid, Private = Private, Self-pay/uninsured $=$ Self-pay, Other = No charge and Other

${ }^{5}$ Metropolitan = large metropolitan and small metropolitan, Non-metropolitan = micropolitan and not metropolitan or micropolitan.

${ }^{6}$ Median income of the zip code reported by quartile: $\mathrm{Q} 1=\$ 1-\$ 38,999, \mathrm{Q} 2=\$ 39,000-\$ 47,999, \mathrm{Q} 3=\$ 48,000-\$ 62,999, \mathrm{Q} 4=\$ 63,000$ or $\mathrm{more}$. 

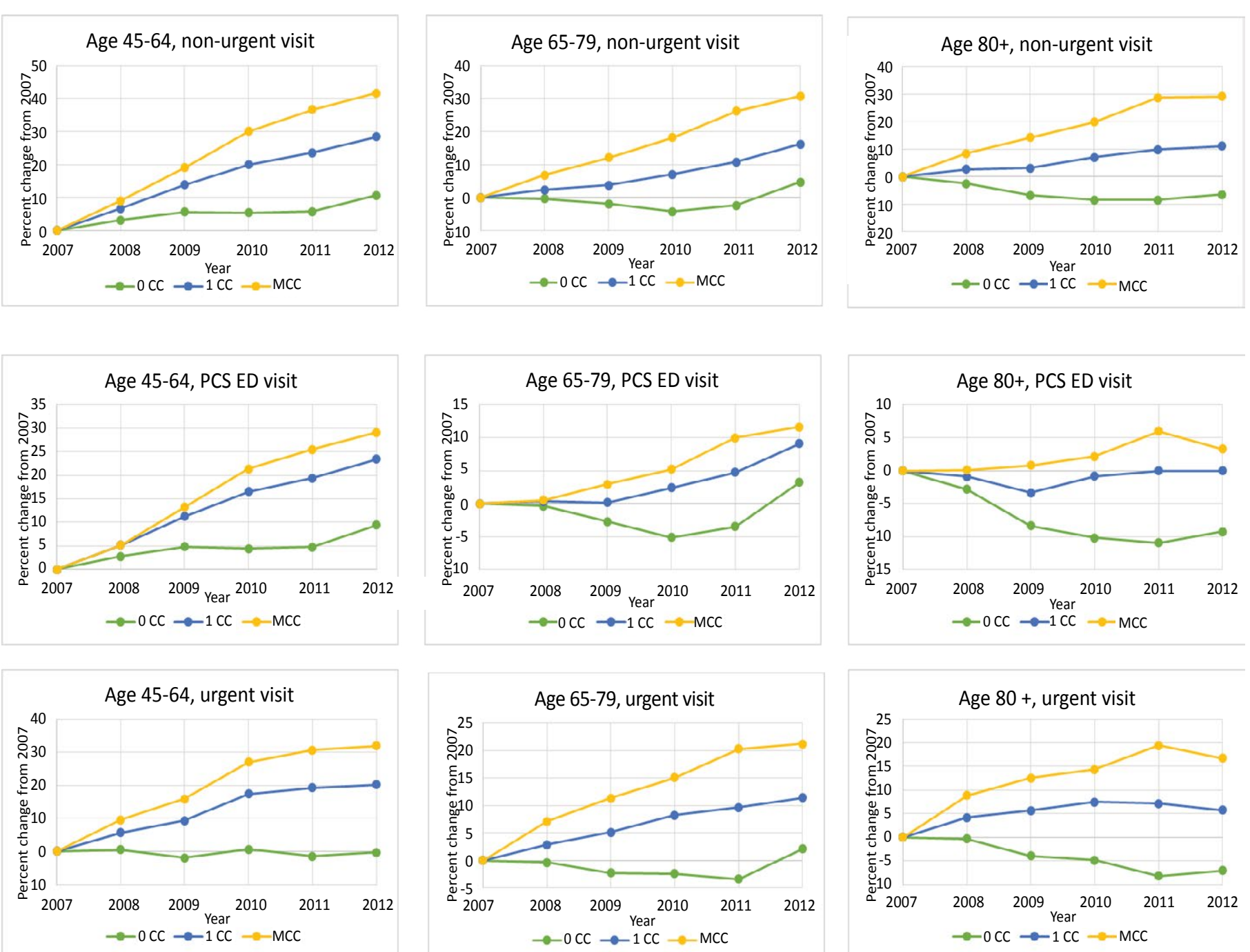

Figure 1 Nonurgent, Primary Care Sensitive, and Urgent visits by Age Category, 2007- 2012. ${ }^{1,2,3}$

${ }^{1}$ Source: Agency for Healthcare Research and Quality (AHRQ), Center for Delivery, Organization, and Markets, Healthcare Cost and Utilization Project (HCUP), Nationwide Emergency Department Sample (NEDS), 2007 and 2012.

${ }^{2}$ Chronic conditions and Clinical Classification Software Codes: hypertension $(98,99)$, coronary heart disease $(100,101)$, stroke $(109-$ $112)$, diabetes $(49,50)$, cancer, excluding non-melanoma skin cancer (11-43), arthritis $(203,203)$, hepatitis (6), chronic kidney disease (158), asthma (128), and chronic obstructive pulmonary disease (COPD) (127).

${ }^{3}$ All ED visits with a probability of "Emergent-ED Care Needed-Not Preventable/Avoidable (ednncpa)" > 0.5 or classified as "injury" were classified "urgent." Those with combined probabilities of "non-emergent (ne)" + "emergent primary care treatable (epct)" > 0.5 were classified as "nonurgent." "Primary care sensitive (PCS)" ED visits included all nonurgent visits defined above (i.e., cases with ne + epct >0.5) and those with a probability of "Emergent-ED Care Needed-Potentially Avoidable (edncpa)" $>0.5$.

*All differences are statistically significant from zero at the $\alpha<0.001$ level.

trend for the 45-64 age group, a U-shaped trend for the 65-79 age group, and a declining trend for the $80+$ age group. Similar patterns were found for urgent ED visits.

As shown in Figure 2, nonurgent, PCS, and urgent ED visits for all three chronic condition categories declined sharply from 2009 to 2010 in non-metropolitan hospitals and increased slightly from 2010 to 2012 across all urgency and chronic condition categories. However, for metropolitan EDs, all three urgency types of ED visits increased across the board for OCC, 1CC, and MCC. A spike of growth in all urgency levels of ED visits from 2009 to 2010 were observed, contrary to the decline in non-metropolitan hospitals during the same period.

\section{Discussion}

Our trend analysis and descriptive results strongly suggest that chronic conditions, especially multiple chronic conditions, played a significant role in the growth of ED visits in general, and in nonurgent and PCS ED visits, in particular. The increases in nonurgent and PCS visits may represent the pressing need, as well as opportunities, to reduce potentially avoidable ED visits 

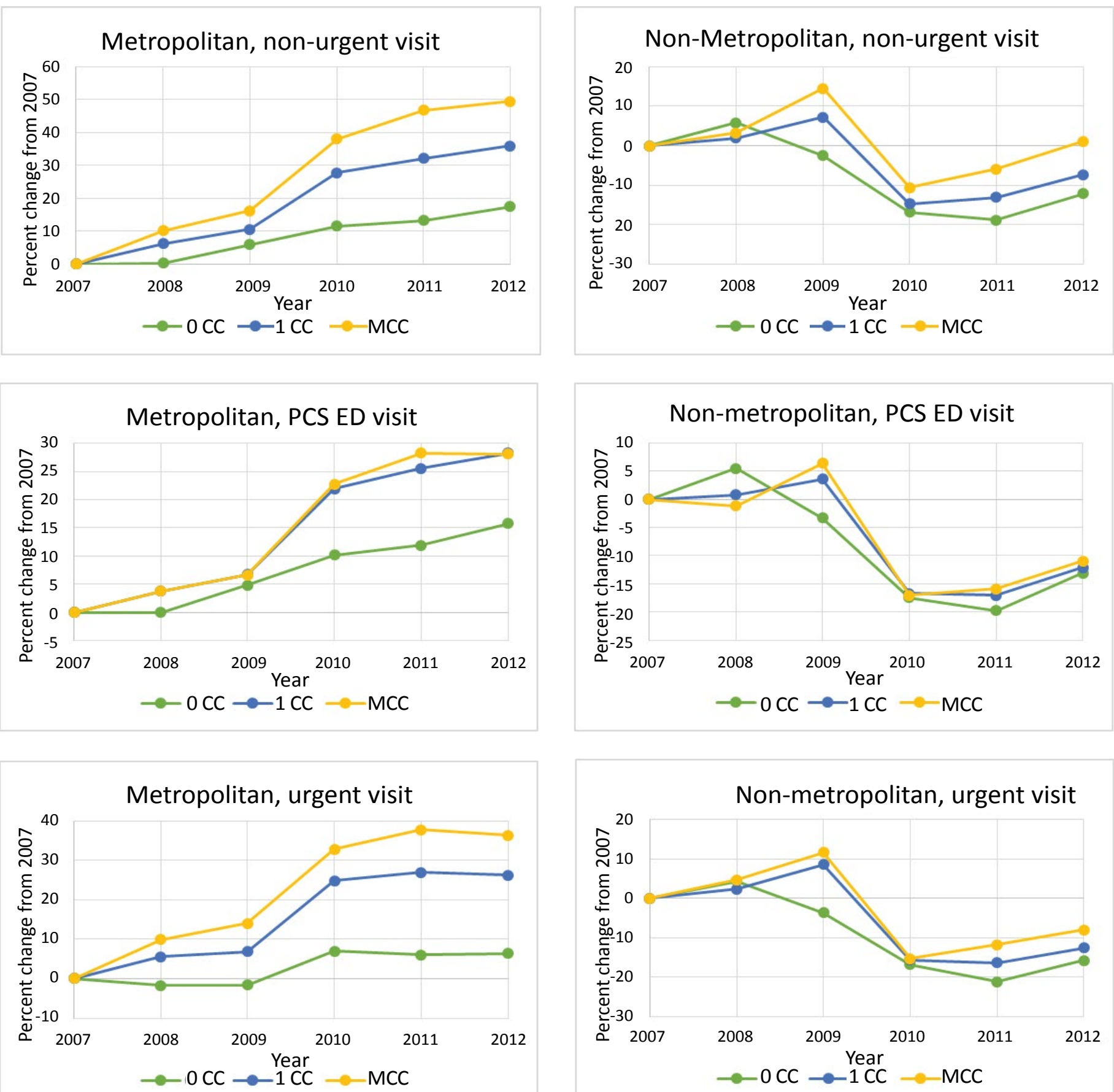

Figure 2

Nonurgent, Primary Care Sensitive, and Urgent Visits by Metropolitan and Non-metropolitan status, 2007-2012. ${ }^{1,2,3,4}$

${ }^{1}$ Source: Agency for Healthcare Research and Quality (AHRQ), Center for Delivery, Organization, and Markets, Healthcare Cost and Utilization Project (HCUP), Nationwide Emergency Department Sample (NEDS), 2007 and 2012.

${ }^{2}$ Chronic conditions and Clinical Classification Software Codes: hypertension (98,99), coronary heart disease $(100,101)$, stroke (109$112)$, diabetes $(49,50)$, cancer, excluding non-melanoma skin cancer (11-43), arthritis $(203,203)$, hepatitis $(6)$, chronic kidney disease (158), asthma (128), and chronic obstructive pulmonary disease (COPD) (127).

${ }^{3}$ All ED visits with a probability of "Emergent-ED Care Needed-Not Preventable/Avoidable (ednncpa)" > 0.5 or classified as "injury" were classified "urgent." Those with combined probabilities of "non-emergent (ne)" + "emergent primary care treatable (epct)" $>0.5$ were classified as "nonurgent." "Primary care sensitive (PCS)" ED visits included all nonurgent visits defined above (i.e., cases with ne + epct $>0.5$ ) and those with a probability of "Emergent-ED Care Needed-Potentially Avoidable (edncpa)" >0.5.

${ }^{4}$ Metropolitan = large metropolitan and small metropolitan, Non-metropolitan = micropolitan and not metropolitan or micropolitan. *All differences are statistically significant from zero at the $\alpha<.001$ level. 
and unnecessary health care costs, especially those associated with visits made by patients with higher illness burdens.

Another implication of our analysis worthy of note is the different role played by age and chronic conditions as a driver of ED visits over time. Perhaps the most interesting finding was that nonurgent visits increased at a higher percentage rate than did PCS and urgent visits in all age categories, with ED visits made by patients with $\mathrm{MCC}$ growing at higher rates than those by patients with OCC and 1CC. Also, while previous studies have shown that the most frequent users of the ED are insured, our trend analysis shows a larger percentage growth among public and self-pay/ uninsured for nonurgent, PCS, and urgent visits. Although in terms of reported number of visits, self-pay/uninsured visits were still the smallest, their growth rate was much higher over time when compared to those made by patients with other types of insurance coverage [3]. Given the age and insurance growth rates, policy measures designed for the Medicare population may not work for the younger age groups in dealing with the problem of overuse of care for those with different chronic disease burdens. Commercial insurance providers and Medicaid will face the burden of paying for chronic conditions. However, we have only used ED data through 2012; it is possible that these trends may not persist once data after 2014 is examined due to Medicaid expansion and the availability of healthcare marketplace plans. Antwi and colleagues found a decrease in ED visit use among young adults following dependent coverage expansion in $\mathbf{2 0 1 0}$ mandated by the ACA, yet they are generally a healthier population with fewer chronic conditions than the age group that we examined [19].

In terms of geographic location, the West and South regions experienced the most growth in ED visits, with the largest growth for $1 \mathrm{CC}$ and MCC urgent, PCS, and nonurgent visits taking place out West, while the largest growth for urgent, nonurgent, and
PCS visits for OCC occurred in the South. Studies have shown that ED patients in California are more seriously ill than other states and that the West has larger rates of substance abuse ED visits than other census regions, which may account for the higher percentage growth of all types of ED visits for those with $1 C C$ and MCC $[20,21]$. A large shift from non-metropolitan EDs to metropolitan EDs across all levels of urgency for all chronic condition categories occurred between 2009 and 2010. Rural hospitals have been closing at an increasing rate since 2008 . Although some rural hospitals closed due to the recession, many more closures (56) occurred after 2010 [22-25]. Older adults have been shown to avoid seeking care due to rural hospital closures, which could exacerbate the problem of increased non-urgent and PCS visits for MCC [26].

Our study has several limitations. First, as a trend analysis, this study provides a descriptive look at ED trends over a six-year period. It did not attempt to test any specific hypothesis or examine the influences of multiple underlying factors responsible for the observed trends. Our future research will examine these trends, controlling for factors that may impact the urgency and frequency of ED use. We also did not examine race, which has often been significantly associated with nonurgent and PCS use of the ED. The NEDS withholds race from its public use files so that the patients whose data were used cannot be identified. We also only examined adults ages 45 and older, due to the low prevalence of chronic conditions in the younger age groups [12]. To our knowledge, this is the first study that examined the burden of multiple chronic conditions by the NHIS MCC classification using the NYU algorithm to identify urgent, PCS, and nonurgent ED visits. Our findings show a large and increasing number of MCC visits across all categories of ED visits. The increase in nonurgent and PCS visits by those with MCC should be of particular concern to ED providers and hospital administrators. 


\section{References}

1 Oster A, Bindman AB (2003) Emergency department visits for ambulatory care sensitive conditions: insights into preventable hospitalizations. Med Care 41: 198-207.

2 Vinton DT, Capp R, Rooks SP, Abbott JT, Ginde A (2014) Frequent users of US emergency departments: characteristics and opportunities for intervention. Emerg Med J 31: 526-532.

3 Miller JB, Brauer E, Rao H, Wickenheiser K, Dev S, et al. (2013) The most frequent ED patients carry insurance and a significant burden of disease. Am J Emerg Med 31: 16-19.

4 Gindi RM, Black LI, Cohen RA (2016) Reasons for Emergency Room Use Among U.S. Adults Aged 18-64: National Health Interview Survey, 2013 and 2014. National Health Statistics Reports 1-16.

5 Chen BK, Hibbert J, Cheng X, Bennett K (2015) Travel distance and sociodemographic correlates of potentially avoidable emergency department visits in California, 2006-2010: an observational study. Int J Equity Health 14: 30.

6 Chen W, Waters TM, Chang CF (2015) Insurance Impact on Nonurgent and Primary care-sensitive emergency department use. Am J Manag Care 21: 210-217.

7 Lee MH, Schuur JD, Zink BJ (2013) Owning the cost of emergency medicine: Beyond 2\%. Ann Emerg Med 62: 498-505.

8 National Priorities Partnership (2010) Reducing Emergency Department Overuse: a \$38 Billion Opportunity. Washington, DC.

9 National Priorities Partnership (2000) National Priorities and Goals: Aligning Our Efforts to Transform America's Healthcare. Washington, DC.

10 Agency for Healthcare Research and Quality (2015) The Nationwide Emergency Department Sample (NEDS) Database Documentation.

11 Freid VM, Bernstein AB, Bush MA (2012) Multiple chronic conditions among adults aged 45 and over: trends over the past 10 years. NCHS Data Brief 100: 1-8.

12 Gerteis J, Izrael D, LeRoy L, Ricciardi R, Miller T, et al. (2010) Multiple Chronic Conditions Chartbook. Agency of Healthcare Research and Quality.

13 Ward BW, Schiller JS (2013) Prevalence of multiple chronic conditions among US adults: estimates from the National Health Interview Survey, 2010. Prev Chronic Dis 10: 1-15.
14 Goodman R A, Posner SF, Huang ES, Parekh AK, Koh HK (2013) Defining and measuring chronic conditions: imperatives for research, policy, program, and practice. Prev Chronic Dis 10: 1-5.

15 Billings J, Parikh N, Mijanovich T (2000) Emergency Department Use in New York City: A Substitute for Primary Care? Issue Brief Commonw Fund 433: 1-5.

16 Gandhi SO, Sabik L (2014) Emergency department visit classification using the NYU algorithm. Am J Manag Care 20: 315-320.

17 Tsai JCH, Chen WY, Liang YW (2011) Nonemergent emergency department visits under the National Health Insurance in Taiwan. Health Policy 100: 189-195.

18 Ballard DW, Price M, Fung V, Brand R (2010) Validation of an algorithm for categorizing the severity of hospital emergency department visits. Med Care 48: 1-15.

19 Antwi YA, Moriya AS, Simon K, Sommers BD (2015) Changes in Emergency Department Use Among Young Adults After the Patient Protection and Affordable Care Act's Dependent Coverage Provision. Ann Emerg Med 65 : 664-672.

20 Larkin GL, Claassen CA, Emond JA, Pelletier AJ, Camargo CA (2005) Trends in U.S. emergency department visits for mental health conditions, 1992 to 2001. Psychiatr Serv 56: 671-677.

21 McConville S, Lee H (2008) Emergency Department Care in California. PPIC.

22 https://www.shepscenter.unc.edu/programs-projects/rural-health/ rural-hospital-closures/

23 National Rural Health Association (2013) Rural Hospital Closures are Decimating Rural Health Care Delivery.

24 Kaufman BG, Thomas SR, Randolph RK, Perry JR, Thompson TW, et al. (2016) The Rising Rate of Rural Hospital Closures. J Rural Heal 32: $35-43$.

25 Thomas SR, Holmes GM, Pink GH (2016) To What Extent do Community Characteristics Explain Differences in Closure among Financially Distressed Rural Hospitals? J Health Care Poor Underserved 27: 194-203.

26 Countouris M, Gilmore S, Yonas M (2014) Exploring the impact of a community hospital closure on older adults: a focus group study. Health Place 26: 143-148. 